This item was submitted to Loughborough's Research Repository by the author.

Items in Figshare are protected by copyright, with all rights reserved, unless otherwise indicated.

\title{
A fault tree analysis strategy using binary decision diagrams
}

PLEASE CITE THE PUBLISHED VERSION

LICENCE

CC BY-NC-ND 4.0

\section{REPOSITORY RECORD}

Reay, Karen A., and J.D. Andrews. 2019. "A Fault Tree Analysis Strategy Using Binary Decision Diagrams". figshare. https://hdl.handle.net/2134/442. 


\title{
A Fault Tree Analysis Strategy Using Binary Decision Diagrams.
}

\author{
Karen A. Reay and John D. Andrews \\ Loughborough University, Loughborough, Leicestershire, LE11 3TU.
}

\begin{abstract}
The use of Binary Decision Diagrams (BDDs) in fault tree analysis provides both an accurate and efficient means of analysing a system. There is a problem however, with the conversion process of the fault tree to the BDD. The variable ordering scheme chosen for the construction of the BDD has a crucial effect on its resulting size and previous research has failed to identify any scheme that is capable of producing BDDs for all fault trees. This paper proposes an analysis strategy aimed at increasing the likelihood of obtaining a BDD for any given fault tree, by ensuring the associated calculations are as efficient as possible. The method implements simplification techniques, which are applied to the fault tree to obtain a set of 'minimal' subtrees, equivalent to the original fault tree structure. BDDs are constructed for each, using ordering schemes most suited to their particular characteristics. Quantitative analysis is performed simultaneously on the set of BDDs to obtain the top event probability, the system unconditional failure intensity and the criticality of the basic events.
\end{abstract}

\section{Introduction}

The Binary Decision Diagram (BDD) method $^{(1)}$ has emerged as an alternative to conventional techniques for performing both qualitative and quantitative analysis of fault trees. BDD's are already proving to be of considerable use in reliability analysis, providing a more efficient means of analysing a system, without the need for the approximations previously used in the traditional approach of Kinetic Tree Theory ${ }^{(2)}$.

The BDD method does not analyse the fault tree directly, but converts the tree to a binary decision diagram, which represents the Boolean equation for the top event. The difficulty, however, lies with the conversion of the tree to the BDD. An ordering of the fault tree variables (basic events) must be chosen and this ordering can have a crucial effect on the size of the resulting BDD; it can mean the difference between a minimal BDD with few nodes, providing an efficient analysis and being able to produce any BDD at all. There is no universal ordering scheme that can be successfully used to produce a minimal BDD for all fault trees; indeed no scheme has been found that will produce a BDD (minimal or otherwise) for some large fault trees. Emphasis in the research has now turned to applying alternative techniques that will increase the likelihood of obtaining a BDD for any given fault tree, by ensuring the associated calculations are as efficient as possible. 
In this paper, an analysis strategy is proposed which implements these requirements. The initial stage combines two simplification techniques that have been shown to be advantageous in the construction of BDDs: Faunet reduction ${ }^{(3)}$, and linear-time modularisation ${ }^{(4)}$. The reduction technique reduces the fault tree to its minimal logic form, whilst modularisation identifies independent subtrees (modules) existing within the tree that can be analysed separately. This results in a set of 'minimal' fault trees, equivalent to the original fault tree structure.

A neural network is used to select the most appropriate ordering scheme ${ }^{(5,6)}$ for each independent module of the fault tree, based upon its individual characteristics. BDDs are obtained for each module in separate computations, culminating in a set of BDDs, which together represent the original system.

Quantitative analysis is performed simultaneously on the set of BDDs to obtain the top event probability, the system unconditional failure intensity and the criticality of the basic events.

Each of these stages is described in more detail in the following sections and demonstrated throughout with the use of an example fault tree.

\section{Simplification of the Fault Tree Structure}

Two pre-processing techniques are applied to the fault tree in order to obtain the smallest possible subtrees, so that the process of constructing the BDDs becomes simple and efficient. The first stage of pre-processing is Faunet reduction, a technique that is used to restructure the fault tree to its most concise form. Once this has been applied however, it is possible to simplify the analysis further by identifying independent subtrees (modules) within the fault tree that can be treated separately. The linear-time algorithm is an extremely efficient method of modularisation and forms the second stage of the fault tree pre-processing. This results in a set of independent fault trees each with the simplest possible structure, which together describe the original system.

\subsection{Faunet Reduction}

FAUNET reduction is a technique that is used to reduce the fault tree to its minimal form, so eliminating any 'noise' from the system, without altering the underlying logic. Its effectiveness has been demonstrated with its application to a large set of fault trees, where it decreased the size of the resulting BDDs by approximately $50 \%$. The method consists of three stages:

\section{- Contraction}

Subsequent gates of the same type are contracted to form a single gate. This gives a fault tree with an alternating sequence of AND gates and OR gates. 


\section{- Factorisation}

Pairs of events that always occur together in the same gate type are identified. They are combined to form a single complex event, which are given a numerical label from 2000 upwards.

\section{- Extraction}

The following two structures are identified and replaced:

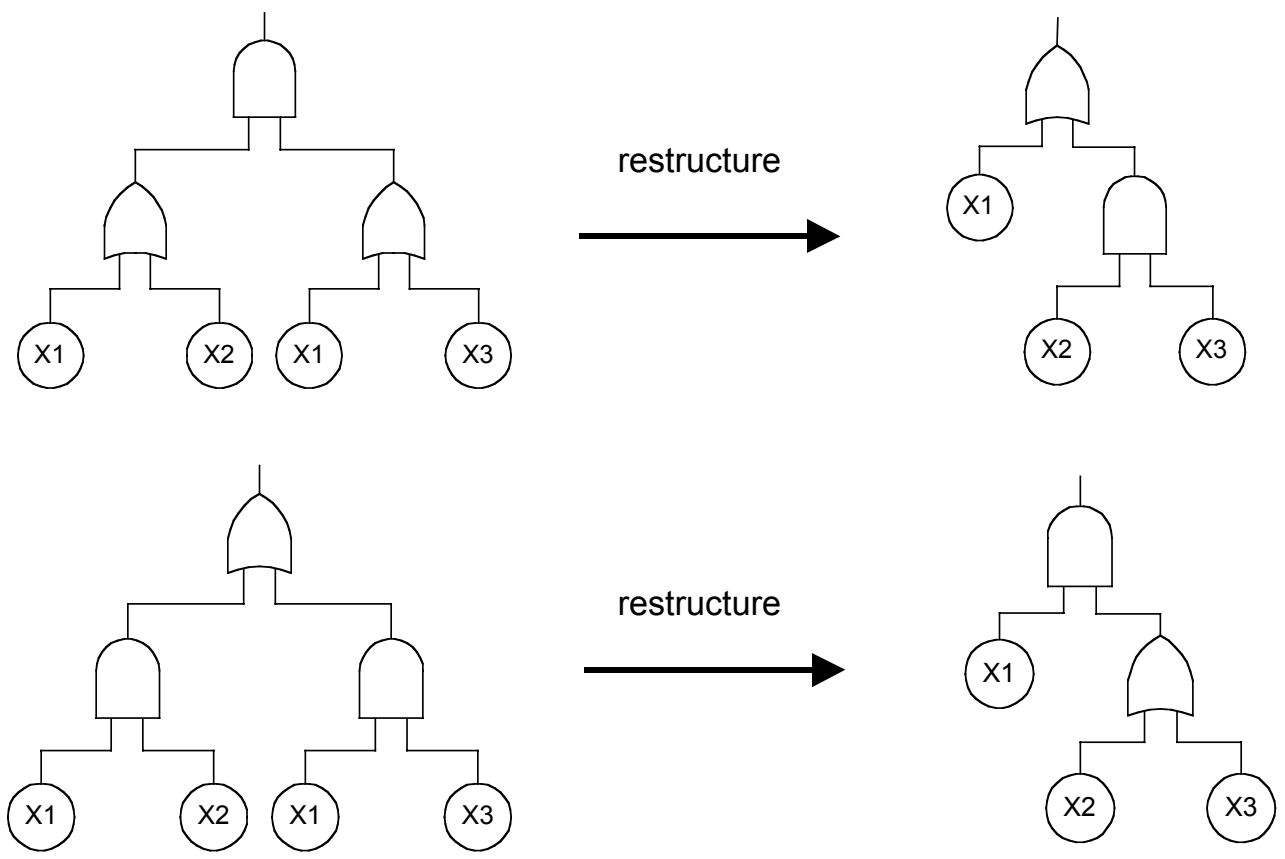

Figure 1: The extraction procedure

The above three steps are repeated until no further changes are possible in the fault tree, resulting in a more compact representation of the system.

For example, consider the fault tree illustrated in Figure 2. 


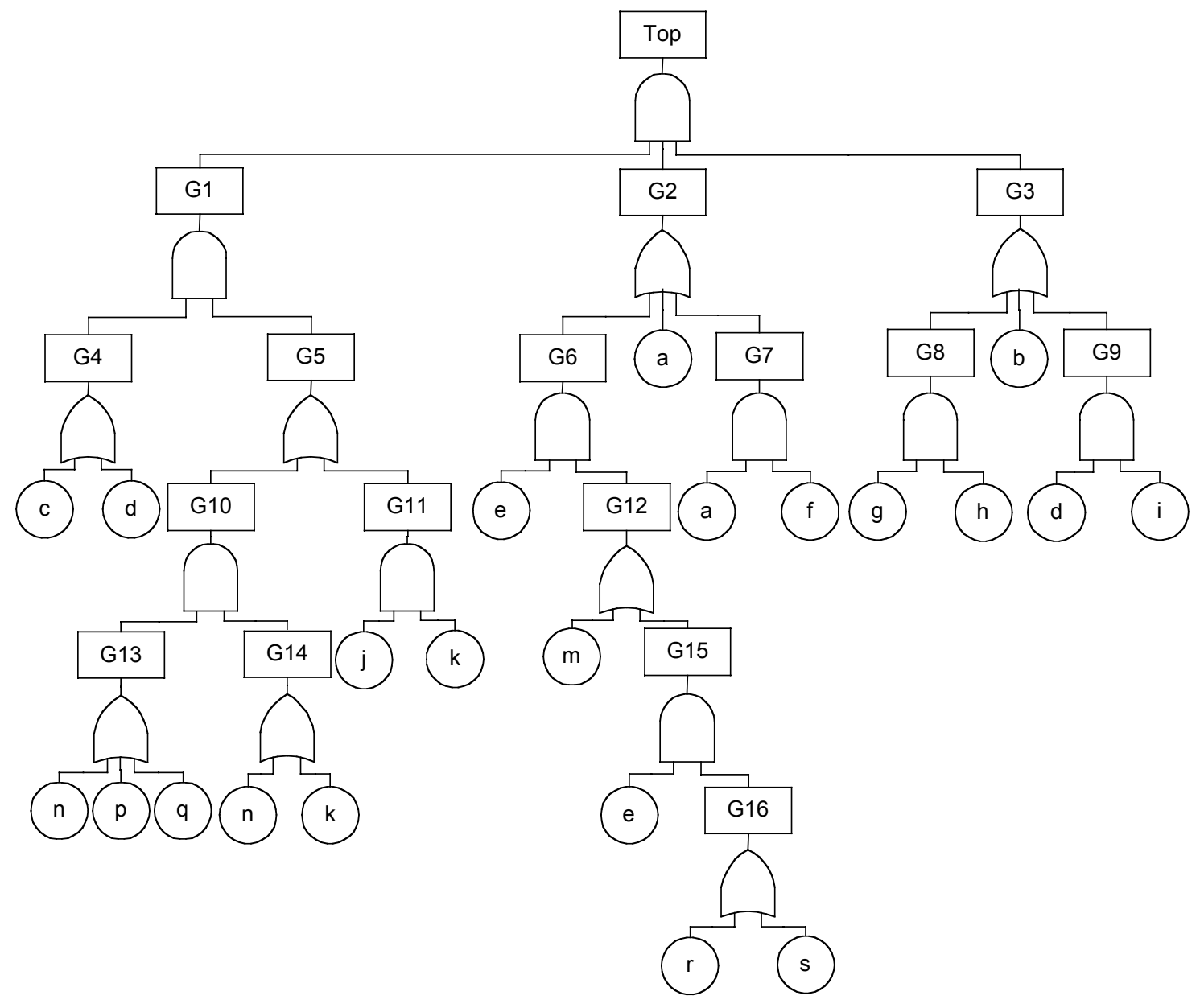

Figure 2: Example fault tree

Upon application of the Faunet reduction technique to this tree, we obtain the much smaller fault tree shown in Figure 3. The corresponding complex event data is shown in Table 1.

\begin{tabular}{|c|c|c|c|}
\hline $\begin{array}{c}\text { Complex } \\
\text { Event, } X_{c}\end{array}$ & $\begin{array}{c}\text { Value of } \\
\text { the gate }\end{array}$ & Event 1 & Event 2 \\
\hline \hline 2000 & AND & $\mathrm{g}$ & $\mathrm{h}$ \\
\hline 2001 & OR & $\mathrm{p}$ & $\mathrm{q}$ \\
\hline 2002 & OR & $\mathrm{r}$ & $\mathrm{s}$ \\
\hline 2003 & OR & 2000 & $\mathrm{~b}$ \\
\hline 2004 & OR & $\mathrm{j}$ & 2001 \\
\hline 2005 & AND & 2004 & $\mathrm{k}$ \\
\hline 2006 & OR & 2005 & $\mathrm{n}$ \\
\hline
\end{tabular}

Table 1: The complex event data after Faunet reduction. 


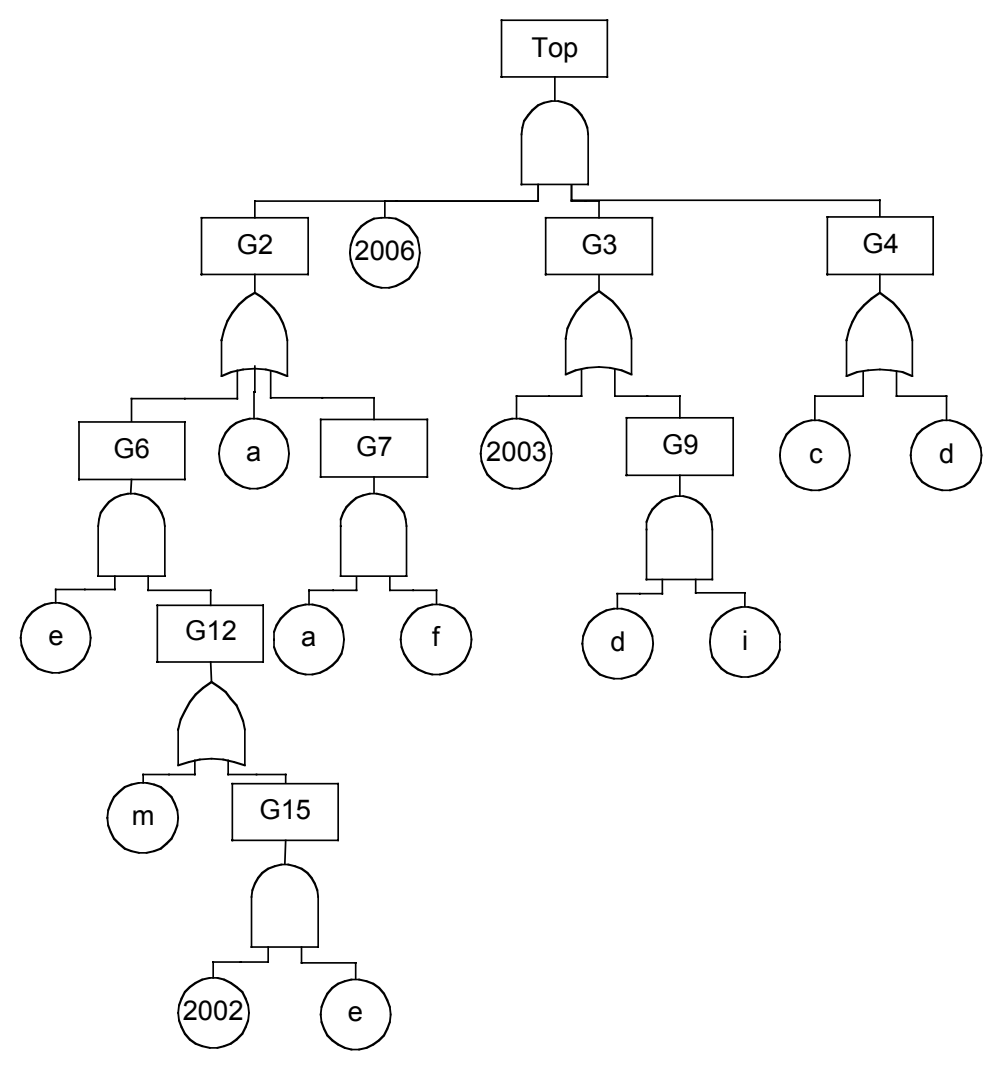

Figure 3: The resulting fault tree after the application of Faunet reduction

Having reduced the fault tree to a more concise form, we now consider the second preprocessing technique of modularisation.

\subsection{Modularisation}

The modularisation procedure does not alter the structure of the tree, but detects modules. A module of a fault tree is a subtree that is completely independent from the rest of the tree. That is, it contains no basic events that appear elsewhere in the fault tree. The advantage of identifying these modules is that each one can be analysed separately from the rest of the tree. The results from subtrees identified as modules are substituted into the higher-level fault trees where the modules occur.

Using the linear-time algorithm, the modules can be identified after two depth-first traversals of the fault tree. The first of these performs a step-by-step traversal recording for each gate and event, the step number at the first, second and final visits to that node. The step number of the second visit to each event is always equivalent to the step number of the first visit to that event. To demonstrate this, refer to the fault tree in Figure 3. Starting at the top event and progressing through the tree in a depth-first manner (and considering the event inputs to a gate before any gate inputs), the gates and events are visited in the order shown in Table 2. Each gate is visited at least twice: once on the way down the tree and again on the way back 
up the tree. Once a gate has been visited, it can be visited again, but the depth-first traversal beneath that gate is not repeated. The step numbers of the visits to the gates and events are shown in Tables 3 and 4.

\begin{tabular}{|c||c|c|c|c|c|c|c|c|c|c|c|}
\hline Step number & 1 & 2 & 3 & 4 & 5 & 6 & 7 & 8 & 9 & 10 & 11 \\
\hline Node & Top & 2006 & G2 & a & G6 & e & G12 & m & G15 & 2002 & e \\
\hline Step number & 12 & 13 & 14 & 15 & 16 & 17 & 18 & 19 & 20 & 21 & 22 \\
\hline Node & G15 & G12 & G6 & G7 & a & f & G7 & G2 & G3 & 2003 & G9 \\
\hline Step number & 23 & 24 & 25 & 26 & 27 & 28 & 29 & 30 & 31 & \\
\hline Node & d & i & G9 & G3 & G4 & c & d & G4 & Top \\
\hline
\end{tabular}

Table 2: Order in which the gates and events are visited in the depth-first traversal of the fault tree in Figure 3.

The second pass through the tree finds the maximum (Max) of the last visits and the minimum (Min) of the first visits of the descendants (any gates and events appearing below that gate in the tree) of each gate; these values are shown in Table 3.

\begin{tabular}{|c||c|c|c|c|c|c|c|c|c|}
\hline Gate & Top & G2 & G3 & G4 & G6 & G7 & G9 & G12 & G15 \\
\hline Visit 1 & 1 & 3 & 20 & 27 & 5 & 15 & 22 & 7 & 9 \\
\hline Visit 2 & 31 & 19 & 26 & 30 & 14 & 18 & 25 & 13 & 12 \\
\hline Last visit & 31 & 19 & 26 & 30 & 14 & 18 & 25 & 13 & 12 \\
\hline Min & 2 & 4 & 21 & 23 & 6 & 4 & 23 & 6 & 6 \\
\hline Max & 30 & 18 & 29 & 29 & 13 & 17 & 29 & 12 & 11 \\
\hline
\end{tabular}

Table 3: Data for the fault tree gates.

\begin{tabular}{|c||c|c|c|c|c|c|c|c|c|c|}
\hline Event & $\mathrm{a}$ & $\mathrm{c}$ & $\mathrm{d}$ & $\mathrm{e}$ & $\mathrm{f}$ & $\mathrm{i}$ & $\mathrm{m}$ & 2002 & 2003 & 2006 \\
\hline Visit 1 & 4 & 28 & 23 & 6 & 17 & 24 & 8 & 10 & 21 & 2 \\
\hline Visit 2 & 4 & 28 & 23 & 6 & 17 & 24 & 8 & 10 & 21 & 2 \\
\hline Last visit & 16 & 28 & 29 & 11 & 17 & 24 & 8 & 10 & 21 & 2 \\
\hline
\end{tabular}

Table 4: Data for the fault tree events.

The principle of the algorithm is that if any descendant of a gate has a first visit step number smaller than the first visit step number of the gate, then it must also occur beneath another gate. Conversely, if any descendant has a last visit step number greater than the second visit step number of the gate, then again it must occur elsewhere in the tree. Therefore, a gate can be identified as heading a module only if: 
- The first visit to each descendant is after the first visit to the gate

and

- The last visit to each descendant is before the second visit to the gate

Therefore, the following gates can be identified as modules:

$$
\text { Top, G2 and G6 }
$$

For completeness, the top event (Top) is included in this list, even though it will always be a module of the fault tree.

The occurrences of these subtrees are replaced by the single modular events, which are named in the same way as complex events (i.e. they take on the next available value above 2000).

$$
\text { Top - 2007, G2 - 2008, G6 - } 2009
$$

Three separate fault trees as shown in Figure 4 now replace the fault tree in Figure 3.

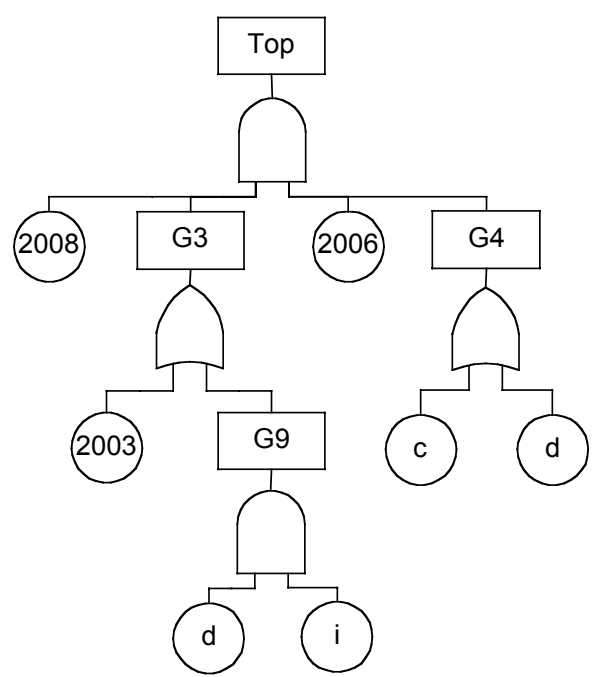

(a) - Module 2007

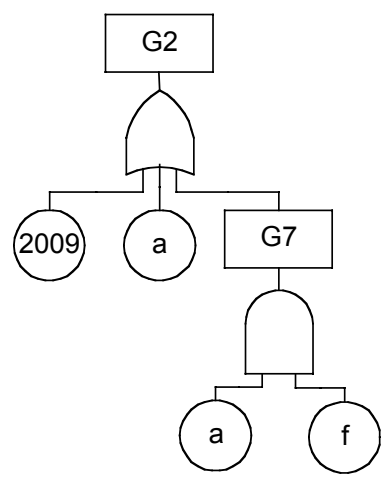

(b) - Module 2008

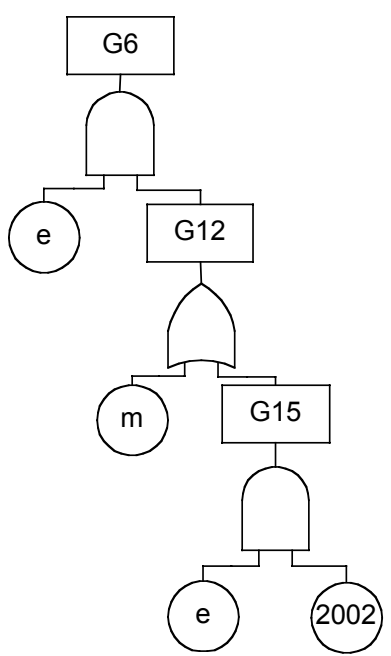

(c) - Module 2009

Figure 4: The three modules obtained from the fault tree shown in Figure 3

Having reduced the fault tree to its minimal form and identified all the independent modules, the pre-processing of the fault tree is complete and the next step is to obtain the associated BDDs.

\section{Obtaining the Associated Binary Decision Diagrams}

A BDD must be constructed for each of the modules. As they all have different properties, using the same variable ordering scheme for each may not be appropriate. Therefore an ordering scheme is selected for each module based on its unique characteristics through the 
use of a pre-programmed neural network. The neural network selects the best ordering scheme from eight possible alternatives, which include both structural and weighted schemes. The BDD for each module is then obtained using the variable ordering determined by the appropriate scheme.

Considering the module '2007' in Figure 4(a), the modified priority depth-first scheme (a depth-first left-right exploration, considering the most repeated events under any gate first and considering gates with only event inputs before any others) was identified as the most suitable by the neural network. This gives the following ordering:

$$
2008<2006<\text { d }<\text { c }<2003<\text { i }
$$

The BDD obtained using this ordering is shown in Figure 5. It is known as the 'primary' BDD, as it represents the top event and is used to calculate the system unavailability.

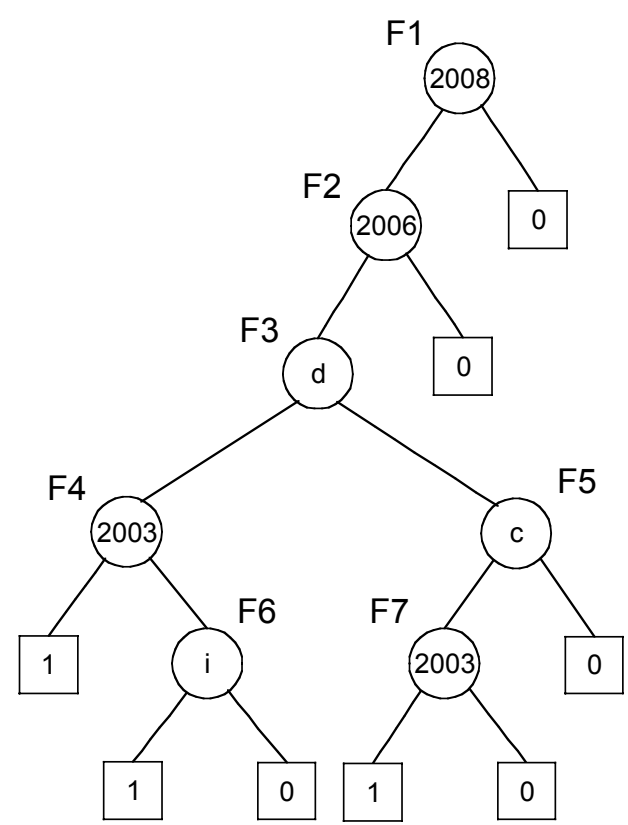

Figure 5: The primary BDD (module '2007') obtained from the ordering $2008<2006<\mathrm{d}<\mathrm{c}<2003<\mathrm{i}$

Each module is treated in the same manner, with its BDD nodes labelled consecutively from the one previously constructed in order to avoid confusion.

The BDDs were constructed for the two remaining modules in the example. The modified depth-first scheme (a depth-first left-right exploration, considering the most repeated events under any gate first) was used for module '2008', producing the ordering:

$a<2009<f$ 
The modified top-down scheme (a left-right, top-down exploration of the tree, considering repeated events first) was used for module '2009' giving:

$\mathrm{e}<\mathrm{m}<2002$

The resulting BDDs, which also illustrate the node labelling, are shown in Figure 6.

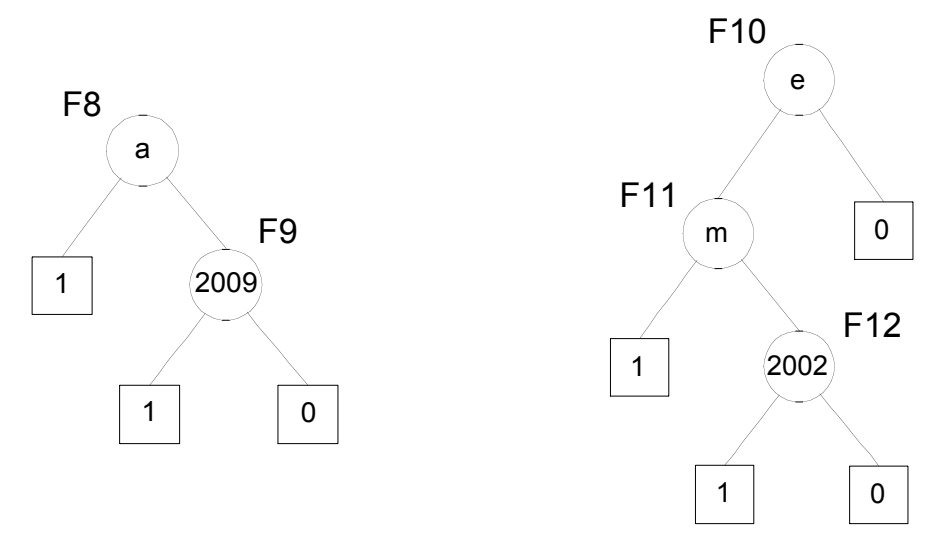
(a) - BDD for module '2008'
(b) BDD for module '2009'

Figure 6: The BDDs for modules '2008' and '2009', demonstrating node labelling

Once the complete set of BDD data has been computed, the quantitative analysis can begin.

\section{Quantitative Analysis}

Quantitative analysis performed on BDDs is an exact and efficient procedure ${ }^{(7)}$, which allows us to determine many properties of the system under consideration. To date, the methods have only been used on BDDs consisting entirely of basic events. As the techniques of reduction and modularisation produce both complex and modular events, the methods need to be extended to consider these extra factors.

The following sections describe the extension of the current methods for dealing with BDDs to those containing complex events and/or modular events. The aim of the analysis is to obtain not only the top event probability and unconditional failure intensity, but to be able to extract the criticality function for the basic events that contribute to the complex events and modules. This is essential, as although we may use reduction and modularisation to help construct the BDDs, we must be able to analyse the system in terms of its original components.

\subsection{System Unavailability}

The probability of occurrence of the top event $\left(Q_{\text {sys }}\right)$ is calculated by summing the probabilities of the disjoint paths through the primary BDD. A depth-first algorithm can perform this calculation very efficiently; further discussion of this procedure can be found in reference 7 . 
The unavailability of each encoded event is required for this calculation. Therefore, the probabilities of the complex and modular events must be obtained from the basic event data.

Determining the unavailability of complex events is straightforward, as they are only a combination of two component events. The calculation depends on whether the events were combined under an AND gate or an OR gate, but if we call the complex event $x_{c}$ and its constituent events $x_{1}$ and $x_{2}$, then we can say:

$$
\begin{aligned}
& \text { AND Gate: } q_{c}=q_{1} q_{2} \\
& \text { OR Gate: } q_{c}=q_{1}+q_{2}-q_{1} q_{2}
\end{aligned}
$$

The probabilities of the complex events are calculated as they are formed, making the process as efficient as possible.

The calculation of the modular events' probabilities is effectively that of finding the probability of occurrence of the 'top event' of each of the modules. Again, a depth-first algorithm is used (as shown in Figure 7), which can repeatedly call itself should further modular events be located within the module itself. Thus, the unavailability of modules encoding only basic and complex events will necessarily be evaluated first.

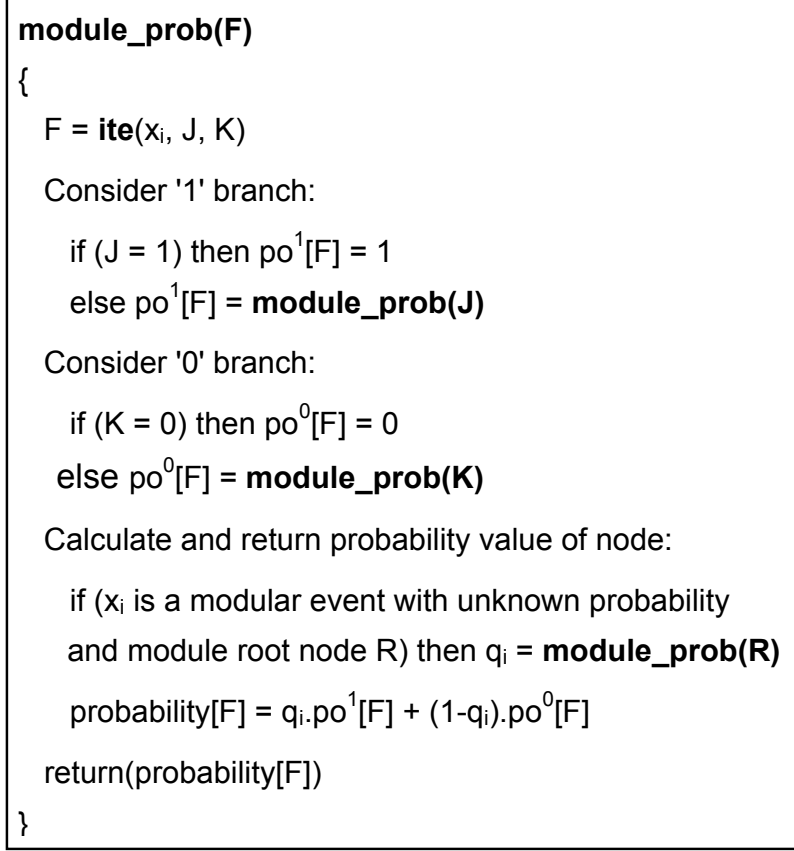

Figure 7: The algorithm for calculating the probability of a module.

Having obtained the probabilities of all complex and modular events, the system unavailability can easily be determined. 


\subsection{System Unconditional Failure Intensity}

The system unconditional failure intensity, $w_{\text {sys }}(t)$, defined as the probability that the top event occurs at $t$ per unit time, is given by:

$$
w_{\text {sys }}(t)=\sum_{i} G_{i}(q(t)) \cdot w_{i}(t)
$$

where $G_{i}(q(t))$ is the criticality function for each component and $\mathrm{w}_{\mathrm{i}}(\mathrm{t})$ is the component unconditional failure intensity

The criticality function is defined as the probability that the system is in a critical state with respect to component $i$ and that the failure of component $i$ would then cause the system to go from a working to a failed state. Therefore:

$$
G_{i}(\mathbf{q}(t))=Q\left(1_{i}, \mathbf{q}(t)\right)-Q\left(0_{i}, \mathbf{q}(t)\right)
$$

where $Q\left(1_{i}, \mathbf{q}(t)\right)$ is the probability of system failure with $q_{i}(t)=1$ and $Q\left(0_{i}, \mathbf{q}(t)\right)$ is the probability of system failure with $\mathrm{q}_{i}(\mathrm{t})=0$.

An efficient method of calculating the criticality function from the $\mathrm{BDD}^{(7)}$ considers the probabilities of the path sections of the BDD up to and after the nodes in question, resulting in the following expression:

$$
G_{i}(q(t))=\sum_{n} p r_{x_{i}}(q(t))\left[p o_{x_{i}}^{1}(q(t))-p o_{x_{i}}^{0}(q(t))\right]
$$

where: $\operatorname{pr}_{x_{i}}(q(t))$ - the probability of the path section from the root vertex to the node $x_{i}$ (set to one for the root vertex) $\mathrm{po}_{\mathrm{x}_{i}}^{1}(\mathbf{q}(\mathrm{t}))$ - the probability of the path section from the '1' branch of node $x_{i}$ to a terminal 1 node $\mathrm{po}_{\mathrm{x}_{i}}^{0}(\mathbf{q}(\mathrm{t}))$ - the probability of the path section from the ' 0 ' branch of node $\mathrm{x}_{\mathrm{i}}$ to a terminal 1 node

$\mathrm{n}$ - $\quad$ all nodes for variable $\mathrm{x}_{\mathrm{i}}$ in the BDD.

For a single BDD encoding only basic events, one pass of the BDD is required to calculate $\mathrm{pr}_{\mathrm{x}_{\mathrm{i}}}(\mathrm{q}), \mathrm{po}_{\mathrm{x}_{\mathrm{i}}}^{1}(\mathrm{q})$ and $\mathrm{po}_{\mathrm{x}_{\mathrm{i}}}^{0}(\mathrm{q})$ for each node (subsequently referred to as the 'path probabilities' of a node), from which the criticality function of each basic event can be determined, leading to the evaluation of the system failure intensity. However, this method does not take account of complex and modular events. It is possible to calculate $\mathrm{w}_{\mathrm{sys}}$ by considering only the events encoded in the primary BDD, but this requires not only the criticality of the modular and complex events but also their failure intensities. Although these are relatively simple to calculate, they are values that have no further use in the analysis. Instead, we calculate the criticality functions of each of the basic events and use these together with their unconditional 
failure intensities to calculate $\mathrm{w}_{\text {sys }}$. This also allows analysis of the contributions to system failure through component or basic event importance measures. $G_{i}(q)$ is Birnbaum's measure of component importance. It is also a major element required to evaluate the criticality measure of component importance.

The criticality functions of the basic events within the primary BDD are still calculated at the end of the analysis once the path probabilities have been found for the nodes of the primary BDD. The calculation of the criticality functions of the basic events incorporated within complex events and modules are described in the following sections.

\subsection{Criticality of Basic Events within Complex Events}

Once the path probabilities are known for a complex event node, the complex event must be further analysed by assigning appropriate values of $\operatorname{pr}_{x_{i}}(q), p o_{x_{i}}^{1}(q)$ and $p o_{x_{i}}^{0}(q)$ to its component events. These are required so that the criticality functions of the basic events can be evaluated. Consider the complex event $X_{c}$, shown in Figure 8.

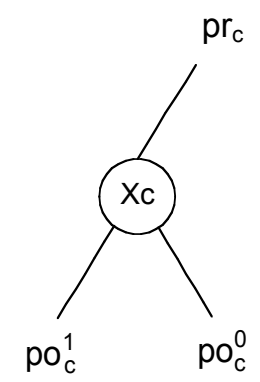

Figure 8: A complex event node within a BDD

The two events that make up this complex event are either joined by an AND gate or an OR gate, which gives the possible ite (if-then-else ${ }^{(1)}$ ) structures and corresponding BDDs as shown in Figure 9.

$$
\begin{aligned}
\text { AND: } & X_{c}=X_{1} \cdot X_{2} \\
& X_{c}=\operatorname{ite}\left(X_{1}, \operatorname{ite}\left(X_{2}, 1,0\right), 0\right)
\end{aligned}
$$

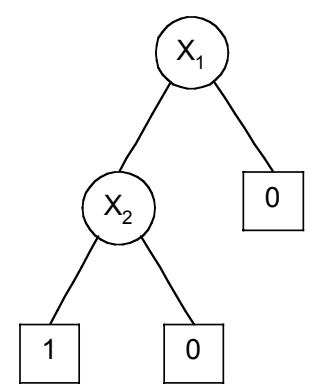

$$
\text { OR: } \begin{aligned}
X_{c} & =X_{1}+X_{2} \\
& X_{c}=\operatorname{ite}\left(X_{1}, 1, \text { ite }\left(X_{2}, 1,0\right)\right)
\end{aligned}
$$

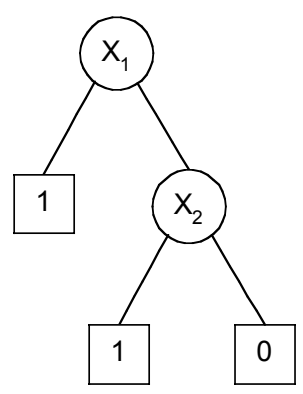

Figure 9: The possible BDD structures of a complex event 
The complex event node effectively replaces one of these structures in the original BDD - this could be either the primary BDD or the BDD of a module. In order to evaluate the path probabilities of the nodes encoding these component events, we simply replace any terminal ' 1 ' branches with the probability of the paths below the ' 1 ' branch of the complex node and the terminal ' 0 ' branches with the probability of the paths below the ' 0 ' branch of the complex node. The probability of the paths before the root vertex does not have the usual value of 1 , but takes on the value of $\operatorname{pr}_{x_{i}}(q)$ of the complex event node. This is shown in Figure 10.

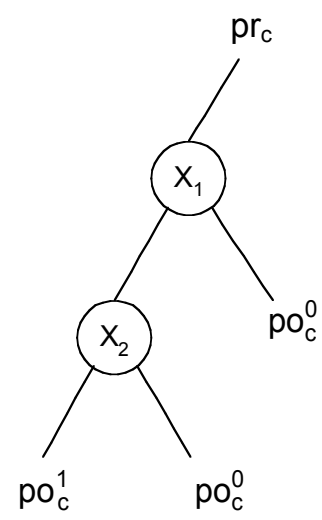

(a) $X_{c}=X_{1} \cdot X_{2}$

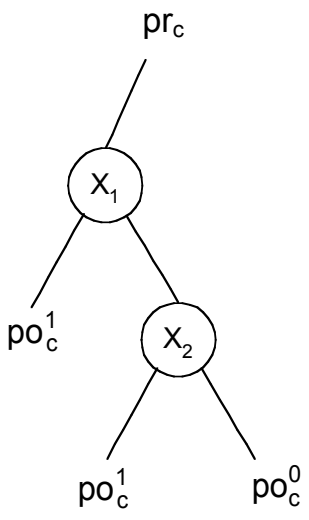

(b) $X_{c}=X_{1}+X_{2}$

Figure 10: The complex event structure

Using Figure 10, we can calculate the values of $\operatorname{pr}_{x_{i}}(q), \operatorname{po}_{x_{i}}^{1}(q)$ and $\operatorname{po}_{x_{i}}^{0}(q)$ for the variables $X_{1}$ and $X_{2}$ :

AND:

$$
\begin{array}{ll}
\mathrm{X}_{1}: \quad \mathrm{pr}_{1}=\mathrm{pr}_{\mathrm{c}} \\
& \mathrm{po}_{1}^{1}=\mathrm{q}_{2} \cdot \mathrm{po}_{\mathrm{c}}^{1}+\left(1-\mathrm{q}_{2}\right) \cdot \mathrm{po}_{\mathrm{c}}^{0} \\
& \mathrm{po}_{1}^{0}=\mathrm{po}_{\mathrm{c}}^{0} \\
\mathrm{X}_{2}: \quad & \mathrm{pr}_{2}=\mathrm{pr}_{\mathrm{c}} \cdot \mathrm{q}_{1} \\
& \mathrm{po}_{2}^{1}=\mathrm{po}_{\mathrm{c}}^{1} \\
& \mathrm{po}_{2}^{0}=\mathrm{po}_{\mathrm{c}}^{0}
\end{array}
$$

(8) $\quad X_{2}:$

OR:

$\mathrm{pr}_{1}=\mathrm{pr}_{\mathrm{c}}$

As the events $X_{1}$ and $X_{2}$ may be either basic events or other complex events, this process is repeated until values have been calculated for all contributing basic events. The criticality functions of the basic events are calculated as they are encountered, using Equation 4. The algorithm implementing this method is shown in Figure 11. 


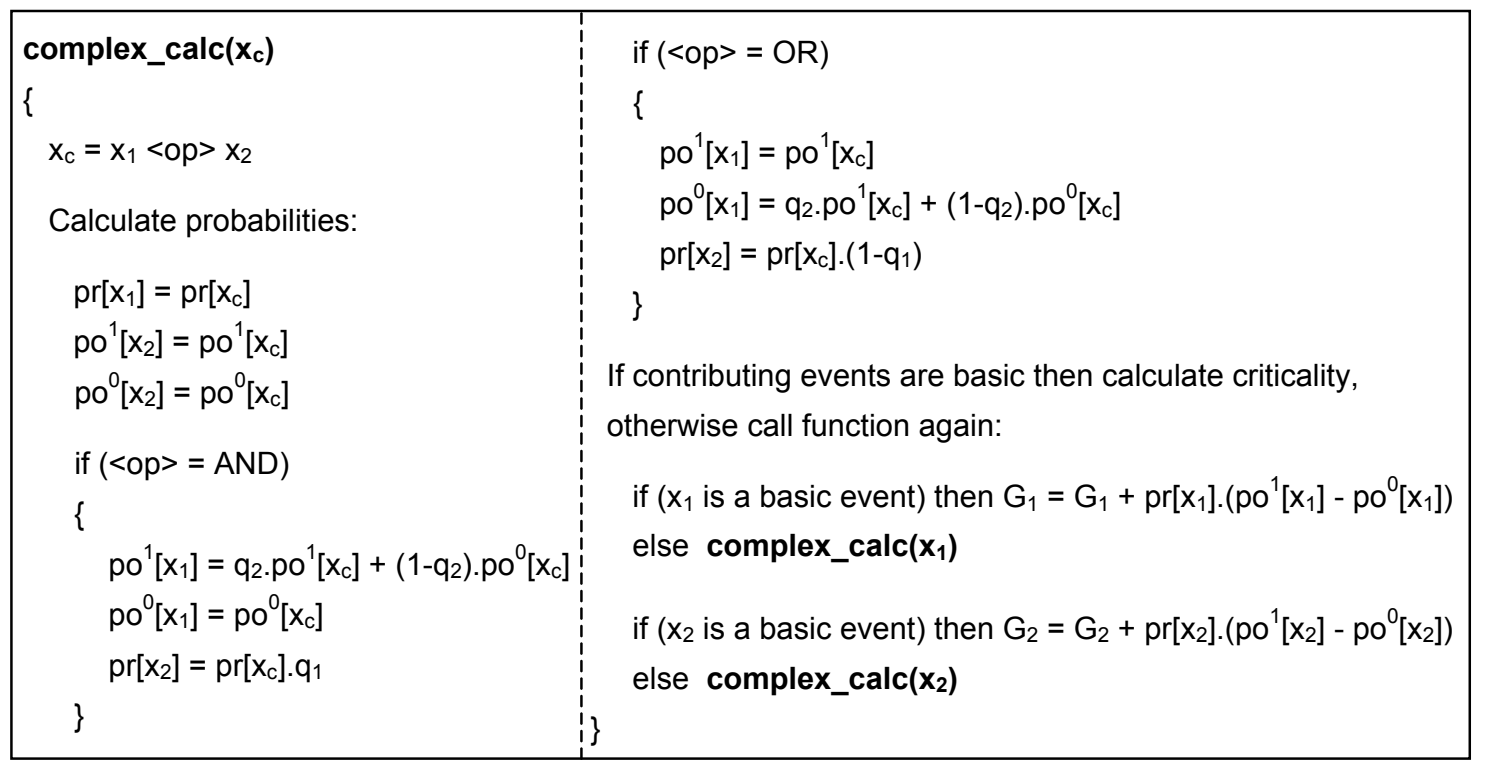

Figure 11: The calculation of the criticality functions of basic events within complex events.

Any complex event may appear more than once in the BDD, resulting in new values of $\mathrm{pr}_{\mathrm{x}_{\mathrm{i}}}(\mathrm{q}), \mathrm{po}_{\mathrm{x}_{\mathrm{i}}}^{1}(\mathrm{q})$ and $\mathrm{po}_{\mathrm{x}_{\mathrm{i}}}^{0}(\mathrm{q})$ being calculated for its component events on each occasion. The criticality function for each of the contributing basic events must be in stages, using the newly assigned values each time. Once this additional criticality value has been calculated for each of the contributing basic events, it is added to the current value so that it is calculated as the analysis proceeds, rather than as a separate procedure at the end of the analysis as is the case for the basic events in the primary BDD.

\subsection{Criticality of Basic Events within Modules}

Modular events are dealt with in a similar way to complex events. Once the path probabilities of the modular event node are known, the module is further analysed to determine the path probabilities of its component nodes. These probabilities must be calculated as they would have been, had the module not been replaced by the single modular event. In order to do this, the values of $\mathrm{po}_{\mathrm{x}_{\mathrm{i}}}^{1}(\mathrm{q})$ and $\mathrm{po}_{\mathrm{x}_{\mathrm{i}}}^{0}(\mathrm{q})$ of the modular event replace the terminal ' 1 ' and ' 0 ' branches, and the probability of the paths before the root vertex of the module is assigned the value of $\operatorname{pr}_{\mathrm{x}_{\mathrm{i}}}(\mathrm{q})$ of the modular event. This is demonstrated in Figure 12. 


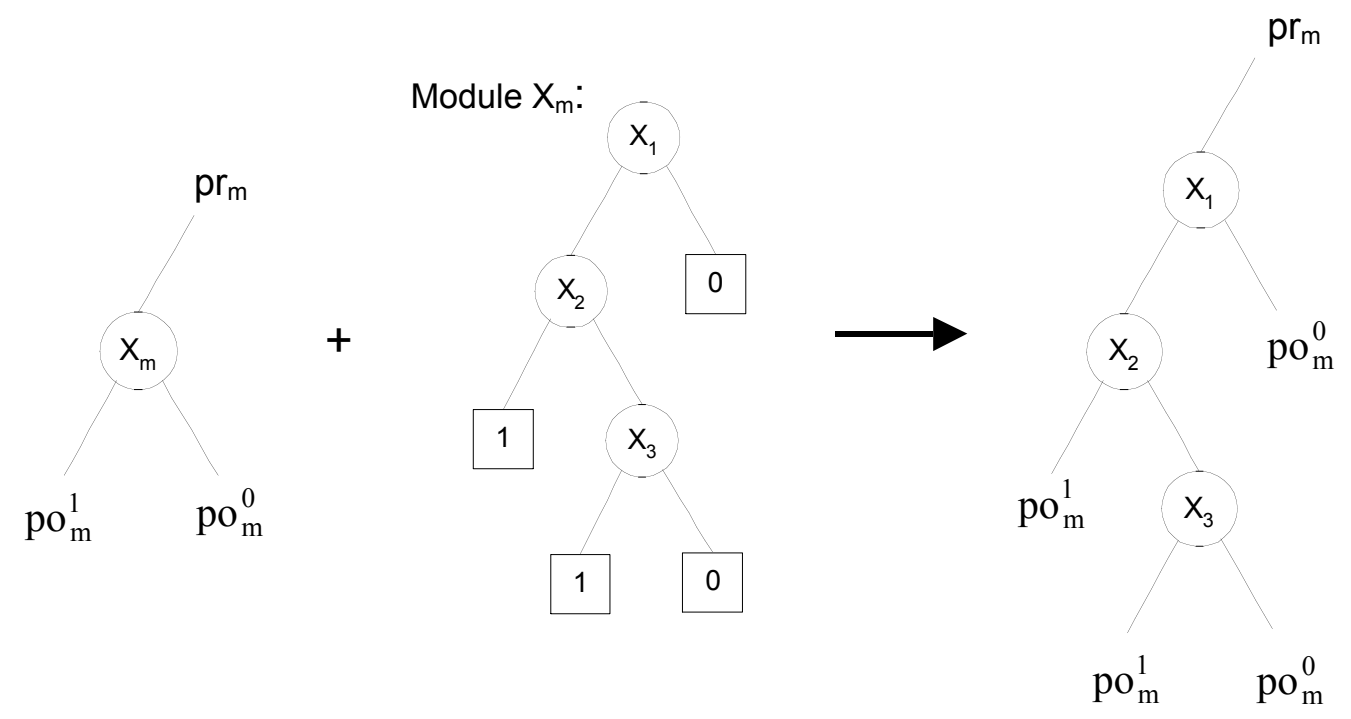

Figure 12: Replacing a modular event with the entire module structure.

Unlike complex events, the structure of modules is not fixed. They can contain any number of events (basic, complex, or indeed other modular events), connected by any number of gates. Therefore, the probabilities are assigned by performing a pass through the whole BDD, a process that is capable of dealing with any structure. The criticality functions of the basic events are then calculated according to equation 4.

As with the complex events, the calculations required to obtain the path probabilities for the nodes within the module must be repeated for each occurrence of the modular event in the BDD. The values are then used to calculate the additional contributions to the criticality functions of the basic events that arise due to the further occurrences of the modular event. This can be seen in the following example.

Having determined the criticality function of each basic event, the system failure intensity can be evaluated using equation 2 and any further importance measure analysis undertaken.

\subsection{Quantitative Analysis Example}

This quantitative analysis can be demonstrated using the set of example BDDs obtained in Section 3. The basic event data is shown in Table 5.

\begin{tabular}{|c||c|c|c|c|c|c|c|c|c|}
\hline Event & $\mathrm{a}$ & $\mathrm{b}$ & $\mathrm{c}$ & $\mathrm{d}$ & $\mathrm{e}$ & $\mathrm{f}$ & $\mathrm{g}$ & $\mathrm{h}$ & $\mathrm{i}$ \\
\hline $\mathrm{q}_{\mathrm{i}}$ & 0.003 & 0.0045 & 0.008 & 0.01 & 0.0035 & 0.0025 & 0.015 & 0.012 & 0.009 \\
\hline $\mathrm{w}_{\mathrm{i}}$ & $1.94 \times 10^{-4}$ & $9.90 \times 10^{-7}$ & $2.15 \times 10^{-6}$ & $1.37 \times 10^{-5}$ & $3.92 \times 10^{-6}$ & $8.50 \times 10^{-7}$ & $2.44 \times 10^{-6}$ & $6.40 \times 10^{-7}$ & $2.27 \times 10^{-6}$ \\
\hline \hline Event & $\mathrm{j}$ & $\mathrm{k}$ & $\mathrm{m}$ & $\mathrm{n}$ & $\mathrm{p}$ & $\mathrm{q}$ & $\mathrm{r}$ & $\mathrm{s}$ \\
\hline $\mathrm{q}_{\mathrm{i}}$ & 0.004 & 0.007 & 0.015 & 0.005 & 0.008 & 0.0065 & 0.012 & 0.006 \\
\hline $\mathrm{w}_{\mathrm{i}}$ & $3.92 \times 10^{-6}$ & $6.22 \times 10^{-5}$ & $8.76 \times 10^{-6}$ & $4.86 \times 10^{-6}$ & $1.12 \times 10^{-4}$ & $9.90 \times 10^{-7}$ & $3.53 \times 10^{-5}$ & $7.86 \times 10^{-6}$ \\
\hline
\end{tabular}

Table 5: Basic event data for the example fault tree. 
The probabilities of the complex events are calculated according to equations $1 \mathrm{a}$ and $1 \mathrm{~b}$, as the complex events are formed. These are shown in Table 6.

\begin{tabular}{|c||c|c|c|c|c|c|c|}
\hline Complex Event, $\mathrm{X}_{\mathrm{c}}$ & 2000 & 2001 & 2002 & 2003 & 2004 & 2005 & 2006 \\
\hline $\begin{array}{c}\text { Unavailability of the } \\
\text { complex event, } \mathrm{q}_{c}\end{array}$ & $1.80 \times 10^{-4}$ & $1.44 \times 10^{-2}$ & $1.79 \times 10^{-2}$ & $4.68 \times 10^{-3}$ & $1.84 \times 10^{-2}$ & $1.29 \times 10^{-4}$ & $5.13 \times 10^{-3}$ \\
\hline
\end{tabular}

Table 6: Complex event data.

The probabilities of occurrence of modules '2008' and '2009' are also needed and are evaluated by calculating the probability of the 'top event' of each module. Considering module '2009', the disjoint paths through the BDD are:
1. e.m
2. e. $\bar{m} .2002$

Therefore the probability of the module is given by:

$$
\begin{aligned}
q_{2009} & =q_{e} \cdot q_{m}+q_{e} \cdot\left(1-q_{m}\right) \cdot q_{2002} \\
& =1.14 \times 10^{-4}
\end{aligned}
$$

Similarly for module '2008', the disjoint paths through the BDD are
1. a
2. $\bar{a} .2009$

which gives:

$$
\begin{aligned}
q_{2008} & =q_{a}+\left(1-q_{a}\right) \cdot q_{2009} \\
& =3.11 \times 10^{-3}
\end{aligned}
$$

Having obtained the probabilities of each of the events within the primary BDD, the top event probability can be calculated. The disjoint paths through the primary BDD are:

1. 2008.2006.d.2003

2. $2008.2006 . d . \overline{2003 . i}$

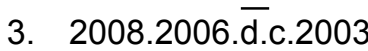

from which we can calculate the system unavailability as:

$$
\begin{aligned}
Q_{\text {sys }} & =q_{2008} \cdot q_{2006} \cdot q_{d} \cdot q_{2003}+q_{2008} \cdot q_{2006} \cdot q_{d} \cdot\left(1-q_{2003}\right) \cdot q_{i}+q_{2008} \cdot q_{2006} \cdot\left(1-q_{d}\right) \cdot q_{c} \cdot q_{2003} \\
& =2.77 \times 10^{-9}
\end{aligned}
$$


The calculations for the system failure intensity start by determining the path probabilities $\mathrm{pr}_{\mathrm{x}_{\mathrm{i}}}(\mathrm{q}), \mathrm{po}_{\mathrm{x}_{\mathrm{i}}}^{1}(\mathrm{q})$ and $\mathrm{po}_{\mathrm{x}_{\mathrm{i}}}^{0}(\mathrm{q})$ for the nodes of the primary BDD. The calculations are shown in Table 7.

\begin{tabular}{|c|c|c|c|c|c|c|}
\hline Node & Variable & $\begin{array}{l}\text { One } \\
\text { branch }\end{array}$ & $\begin{array}{l}\text { Zero } \\
\text { branch }\end{array}$ & $\mathrm{pr}_{\mathrm{x}_{\mathrm{i}}}(\mathrm{q})$ & $\mathrm{po}_{\mathrm{x}_{\mathrm{i}}}^{1}(\mathrm{q})$ & $p o_{x_{i}}^{0}(q)$ \\
\hline $\mathrm{F} 1$ & 2008 & $\mathrm{~F} 2$ & 0 & 1.0 & $\begin{array}{c}\mathrm{q}_{2006} \mathrm{po}^{1}[\mathrm{~F} 2]+\left(1-\mathrm{q}_{2006}\right) \\
\mathrm{po}^{0}[\mathrm{~F} 2]=8.89 \times 10^{-7}\end{array}$ & 0.0 \\
\hline $\mathrm{F} 2$ & 2006 & F3 & 0 & $\begin{array}{c}\operatorname{pr}[\mathrm{F} 1]^{*} \mathrm{q}_{2008}= \\
3.11 \times 10^{-3}\end{array}$ & $\begin{array}{c}\mathrm{q}_{\mathrm{d}} \cdot \mathrm{po}^{1}[\mathrm{~F} 3]+\left(1-\mathrm{q}_{\mathrm{d}}\right) . \\
\mathrm{po}^{0}[\mathrm{~F} 3]=1.73 \times 10^{-4}\end{array}$ & 0.0 \\
\hline F3 & $d$ & $\mathrm{~F} 4$ & F5 & $\begin{array}{c}\operatorname{pr}[F 2]^{*} q_{2006}= \\
1.60 \times 10^{-5}\end{array}$ & $\begin{array}{c}\mathrm{q}_{2003} \cdot \mathrm{po}^{1}[\mathrm{~F} 4]+\left(1-\mathrm{q}_{2003}\right) \\
\mathrm{po}^{0}[\mathrm{~F} 4]=1.36 \times 10^{-2}\end{array}$ & $\begin{array}{l}\mathrm{q}_{\mathrm{c}} \cdot \mathrm{po}^{1}[\mathrm{~F} 5]+\left(1-\mathrm{q}_{\mathrm{c}}\right) . \\
\mathrm{po}^{0}[\mathrm{~F} 5]=3.74 \times 10^{-5}\end{array}$ \\
\hline F4 & 2003 & 1 & F6 & $\begin{array}{l}\operatorname{pr}[\mathrm{F} 3]^{*} \mathrm{q}_{d}= \\
1.60 \times 10^{-7}\end{array}$ & 1.0 & $\begin{array}{c}\text { qi.po }^{1}[\mathrm{~F} 6]+\left(1-\mathrm{q}_{\mathrm{i}}\right) \text {. } \\
\mathrm{po}^{0}[\mathrm{~F} 6]=9.00 \times 10^{-3}\end{array}$ \\
\hline F5 & C & F7 & 0 & $\begin{array}{c}\operatorname{pr}[F 3] .\left(1-q_{d}\right)= \\
1.58 \times 10^{-5}\end{array}$ & $\begin{array}{c}\mathrm{q}_{2003 .} \mathrm{po}^{1}[\mathrm{~F} 7]+\left(1-\mathrm{q}_{2003}\right) \\
\text { po }^{0}[\mathrm{~F} 7]=4.68 \times 10^{-3}\end{array}$ & 0.0 \\
\hline F6 & $\mathrm{i}$ & 1 & 0 & $\begin{array}{l}\operatorname{prF} 4^{*}\left(1-q_{2003}\right) \\
=1.59 \times 10^{-7}\end{array}$ & 1.0 & 0.0 \\
\hline F7 & 2003 & 1 & 0 & $\begin{array}{l}\operatorname{pr}[\mathrm{F} 5] \cdot \mathrm{q}_{\mathrm{c}}= \\
1.26 \times 10^{-7}\end{array}$ & 1.0 & 0.0 \\
\hline
\end{tabular}

Table 7: Results of the quantitative analysis applied to the primary BDD.

The values of $\operatorname{pr}_{x_{\mathrm{i}}}(q), \mathrm{po}_{\mathrm{x}_{\mathrm{i}}}^{1}(q)$ and $\mathrm{po}_{\mathrm{x}_{\mathrm{i}}}^{0}(\mathrm{q})$ for the basic events within the complex events can be calculated according equations 5 - 16. Dealing with the first occurrence of the complex event '2003' at node F4, it can be expanded in terms of its basic events to give the values shown in Table 8 . The criticality functions of the basic events ' $b$ ', ' $g$ ' and ' $h$ ' can be evaluated at this stage and are also shown in Table 8.

\begin{tabular}{|c|c|c|c|c|c|c|}
\hline \multirow{2}{*}{$\begin{array}{l}\text { Complex } \\
\text { event, } X_{C}\end{array}$} & \multirow{2}{*}{$\begin{array}{l}\text { Gate } \\
\text { type }\end{array}$} & \multirow{2}{*}{$\begin{array}{c}\text { Component } \\
\text { event }\end{array}$} & $\mathrm{pr}_{\mathrm{x}_{\mathrm{i}}}(\mathrm{q})$ & $\mathrm{po}_{\mathrm{r}}^{1}(\mathrm{q})$ & $\mathrm{po}_{\mathrm{x}_{\mathrm{i}}}^{0}(\mathrm{q})$ & Criticality \\
\hline & & & \multicolumn{4}{|c|}{ of the component event } \\
\hline \multirow[t]{2}{*}{2003} & OR & $X_{1}=2000$ & $\mathrm{pr}_{2003}=1.60 \times 10^{-7}$ & $\mathrm{po}_{2003}^{1}=1.0$ & $\begin{array}{c}q_{\mathrm{b}} \cdot \mathrm{po}_{2003}{ }_{200}+\left(1-\mathrm{q}_{\mathrm{b}}\right) . \\
\mathrm{po}^{0}{ }_{2003}=1.35 \times 10^{-2}\end{array}$ & - \\
\hline & & $X_{2}=b$ & $\begin{array}{c}\operatorname{pr}_{2002 \cdot}\left(1-\mathrm{q}_{2000}\right)= \\
1.60 \times 10^{-7}\end{array}$ & $\mathrm{po}_{2003}{ }_{200}=1.0$ & $\mathrm{po}_{2003}^{0}=9.00 \times 10^{-3}$ & $1.58 \times 10^{-7}$ \\
\hline \multirow[t]{2}{*}{2000} & AND & $X_{1}=g$ & $\mathrm{pr}_{2000}=1.60 \times 10^{-7}$ & $\begin{array}{l}q_{h} \cdot p^{1}{ }_{2000}+\left(1-q_{h}\right) . \\
\text { po }^{0}{ }_{2000}=2.53 \times 10^{-2}\end{array}$ & po $^{0}{ }_{2000}=1.35 \times 10^{-2}$ & $1.89 \times 10^{-9}$ \\
\hline & & $\mathrm{X}_{2}=\mathrm{h}$ & $\begin{array}{l}\mathrm{pr}_{2000} \cdot \mathrm{qg}_{\mathrm{g}}= \\
2.40 \times 10^{-9}\end{array}$ & po $^{1}{ }_{2000}=1.0$ & po $^{0}{ }_{2000}=1.35 \times 10^{-2}$ & $2.36 \times 10^{-9}$ \\
\hline
\end{tabular}

Table 8: Calculating the criticality functions of the basic events within event '2003'. 
The calculations are repeated for the second occurrence of this complex event at node F7. This results in additional criticality values for the basic events which are added together to give the total criticality function:

$$
\begin{aligned}
& G_{b}=1.58 \times 10^{-7}+1.26 \times 10^{-7}=2.85 \times 10^{-7} \\
& G_{g}=1.89 \times 10^{-9}+1.51 \times 10^{-9}=3.40 \times 10^{-9} \\
& G_{h}=2.36 \times 10^{-9}+1.89 \times 10^{-9}=4.25 \times 10^{-9}
\end{aligned}
$$

The complex event 2006 appears only once in the primary BDD, and expanding it out in terms of its basic events gives the following criticality functions:

$$
G_{j}=3.71 \times 10^{-9}, G_{k}=9.88 \times 10^{-9}, G_{n}=5.40 \times 10^{-7}, G_{p}=3.72 \times 10^{-9}, G_{q}=3.72 \times 10^{-9}
$$

Module 2008, which is encoded in node $\mathrm{F} 1$, is analysed to obtain the path probabilities of its component nodes. The probabilities $\mathrm{po}_{\mathrm{x}_{\mathrm{i}}}^{1}(\mathrm{q})$ and $\mathrm{po}_{\mathrm{x}_{\mathrm{i}}}^{0}(\mathrm{q})$ of the modular event $\left(8.89 \times 10^{-7}\right.$

\begin{tabular}{|c|c|c|c|c|c|c|c|}
\hline Node & Variable & $\begin{array}{l}\text { One } \\
\text { branch }\end{array}$ & $\begin{array}{l}\text { Zero } \\
\text { branch }\end{array}$ & $\mathrm{pr}_{\mathrm{x}_{\mathrm{i}}}(\mathrm{q})$ & $\mathrm{po}_{x_{i}}^{1}(\mathrm{q})$ & $\mathrm{po}_{\mathrm{x}_{\mathrm{i}}}^{0}(\mathrm{q})$ & Criticality \\
\hline F8 & a & 1 & F9 & $\operatorname{pr}[\mathrm{F} 1]=1.0$ & $\begin{array}{l}\text { po }^{1}[\mathrm{~F} 1]= \\
8.89 \times 10^{-7}\end{array}$ & $\begin{array}{c}q_{2009 \cdot p o}[\mathrm{FP}]+ \\
\left(1-\mathrm{q}_{2000}\right) \cdot \mathrm{po}^{0}[\mathrm{Fg}] \\
=1.02 \times 10^{-10}\end{array}$ & $8.89 \times 10^{-7}$ \\
\hline F9 & 2009 & 1 & 0 & $\begin{array}{l}\mathrm{pr}[\mathrm{F} 8]^{*} \mathrm{q}_{\mathrm{a}}= \\
3.00 \times 10^{-3}\end{array}$ & $\begin{array}{c}\mathrm{po}^{1}[\mathrm{~F} 1]= \\
8.89 \times 10^{-7}\end{array}$ & $\mathrm{po}^{0}[\mathrm{~F} 1]=0.0$ & - \\
\hline
\end{tabular}
and 0.0 respectively), replace the terminal ' 1 ' and ' 0 ' branches and the value $\operatorname{pr}_{x_{i}}(q)$ of the modular event is assigned to the module's root vertex. The resultant calculations are shown in Table 9.

Table 9: Results of the quantitative analysis applied to module '2008'.

\begin{tabular}{|c|c|c|c|c|c|c|c|}
\hline Node & Variable & $\begin{array}{l}\text { One } \\
\text { branch }\end{array}$ & $\begin{array}{l}\text { Zero } \\
\text { branch }\end{array}$ & $\mathrm{pr}_{\mathrm{x}_{\mathrm{i}}}(\mathrm{q})$ & $\mathrm{po}_{\mathrm{x}_{\mathrm{i}}}^{1}(\mathrm{q})$ & $\mathrm{po}_{\mathrm{x}_{\mathrm{i}}}^{0}(\mathrm{q})$ & Criticality \\
\hline F10 & e & F11 & 0 & $\begin{array}{c}\operatorname{pr}[\mathrm{FP}]= \\
3.00 \times 10^{-3}\end{array}$ & $\begin{array}{l}q_{m} \cdot p o^{1}[\mathrm{~F} 11]+ \\
\left(1-q_{m}\right) \cdot p 0^{0}[\mathrm{~F} 12] \\
=2.90 \times 10^{-8}\end{array}$ & $\mathrm{po}^{0}[\mathrm{~F} 9]=0.0$ & $8.71 \times 10^{-11}$ \\
\hline F11 & $\mathrm{m}$ & 1 & F12 & $\begin{array}{c}\operatorname{pr}[\mathrm{F} 10] \cdot \mathrm{q}_{\mathrm{e}}= \\
1.05 \times 10^{-5}\end{array}$ & $\begin{array}{c}\text { po }^{1}[\mathrm{~F} 9]=8.89 \\
x 10^{-7}\end{array}$ & $\begin{array}{c}\mathrm{q}_{2002 .} \cdot \mathrm{po}^{1}[\mathrm{~F} 12]+ \\
\left(1-\mathrm{q}_{2002}\right) \cdot \mathrm{po}^{0}[\mathrm{~F} 12] \\
=1.59 \times 10^{-8}\end{array}$ & $9.17 \times 10^{-12}$ \\
\hline $\mathrm{F} 12$ & 2002 & 1 & 0 & $\operatorname{pr}[\mathrm{F} 11] . \mathrm{qm}_{\mathrm{m}}=$ & $\begin{array}{c}\mathrm{po}^{1}[\mathrm{~F} 9]=8.89 \\
\times 10^{-7}\end{array}$ & $\mathrm{po}^{0}[\mathrm{F9}]=0.0$ & - \\
\hline
\end{tabular}

Node F9 encodes another module '2009', which must also be analysed in terms of its basic events. The path probabilities are calculated for each node giving the results shown in Table 10.

Table 10: Results of the quantitative analysis applied to module '2009'. 
The complex event 2002 is expanded out in terms of its basic events to obtain the criticality functions:

$$
\mathrm{G}_{\mathrm{r}}=1.39 \times 10^{-13}, \mathrm{G}_{\mathrm{s}}=1.38 \times 10^{-13}
$$

The only criticality functions that remain to be calculated are those for the basic events within the primary BDD:

$$
\mathrm{G}_{\mathrm{c}}=7.40 \times 10^{-8}, \mathrm{G}_{\mathrm{d}}=2.17 \times 10^{-7}, \mathrm{G}_{\mathrm{i}}=1.59 \times 10^{-7}
$$

The system failure intensity is calculated according to Equation 2 using the basic events' failure intensities and criticality functions to give:

$$
W_{\text {sys }}=1.80 \times 10^{-10}
$$

\section{Conclusions}

This paper has introduced an analysis strategy for dealing with the efficient construction of BDDs from fault trees. The resulting BDDs can encode both complex and modular events, for which the necessary quantitative analysis has been developed. It has also been shown how the analysis proceeds to enable the calculation of the top event probability and the system unconditional failure intensity. In addition, a method to extract the criticality functions for the basic events, which are constituents of both complex events and modules, has been developed. This enables the system to be analysed in terms of its original components. Further quantitative analysis is possible; the methods could be extended to include the calculation of other importance measures for the basic events.

\section{References}

1. Rauzy, A. "New Algorithms for Fault Tree Analysis", Reliab. Engng. Syst. Safety, 40, pp203-211, 1993

2. Vesely, W. E., "A Time Dependent Methodology for Fault Tree Evaluation", Nuclear Eng and Des, 13, pp337-360, 1970

3. Platz, O. and Olsen J. V. "FAUNET: A Program Package for Evaluation of Fault Trees and Networks", Research Establishment Rise Report No 348, DK-4000 Roskilde, Denmark, Sept. 1976

4. Dutuit, Y. and Rauzy, A. "A Linear-Time Algorithm to find Modules of Fault Trees", IEEE Trans. Reliability, 45, No. 3, 1996

5. Bartlett, L. M. "Variable Ordering Heuristics for Binary Decision Diagrams", Doctoral Thesis, Loughborough University, 2000 
6. Bartlett, L. M and Andrews, J. D. "Selecting an Ordering Heuristic for the Fault Tree Binary Decision Diagram Conversion Process using Neural Networks", accepted for Publication in IEEE Trans. Reliability.

7. Sinnamon, R. M. and Andrews, J. D. "Quantitative Fault Tree Analysis using Binary Decision Diagrams", Jour. Europ—en des Systemes Automatis—s, 30, 1996 